Revista de MatemáticA: TeOría y APliCACiones 2015 22(2) : 223-254

CIMPA - UCR ISSN: 1409-2433 (PRINT), 2215-3373 (ONLINE)

\title{
RESULTS ON THE UNIQUENESS OF DIFFERENCE POLYNOMIALS OF ENTIRE FUNCTIONS*
}

\section{RESULTADOS DE UNICIDAD DE DIFERENCIA DE POLINOMIOS DE FUNCIONES ENTERAS}

\author{
HUA WANG ${ }^{\dagger} \quad$ HONG-YAN XU ${ }^{\ddagger}$ \\ Received: 12/May/2014; Revised: 9/Mar/2015; \\ Accepted: 6/May/2015
}

\footnotetext{
* This work was supported by NSFC (Grant No.11301233 and No.61202313), the Natural Science Foundation of Jiangxi Province in China (20132BAB211001,20151BAB201008), and the Foundation of Education Department of Jiangxi (GJJ14644) of China.

${ }^{\dagger}$ Department of Informatics and Engineering, Jingdezhen Ceramic Institute, Jingdezhen, Jiangxi, 333403, China. E-Mail: hhhlucy2012@126.com

${ }^{\ddagger}$ (Corresponding author). Misma dirección que/same address as: H. Wang. E-Mail: xhyhhh@126.com
} 


\begin{abstract}
In this paper, we study the uniqueness of two difference polynomials of entire functions sharing one value, polynomial and small function. Our results of this paper are improvement of the previous theorems given by Chen and Chen [2], Liu, Liu and Cao [22] and Li et al. [19].
\end{abstract}

Keywords: uniqueness; entire function; difference polynomial.

\title{
Resumen
}

En este artículo, se estudia la unicidad de dos polinomios de diferencias de funciones enteras que comparten una función pequeña y polinomial. Nuestros resultados mejoran teoremas previos dados por Chen y Chen [2], Liu, Liu y Cao [22], y Li et al. [19].

Palabras clave: unicidad; función entera; polinomio de diferencias.

Mathematics Subject Classification: 39A70, 30D35.

\section{Introduction and main results}

In this paper, we mainly study the uniqueness of complex difference polynomials of entire functions sharing one value, polynomial and small function with finite weight. The fundamental results and the standard basics of the Nevanlinna value distribution theory of meromorphic functions are used(see [10, 30, 31]). A meromorphic function $f$ means meromorphic in the complex plane. If no poles occur, then $f$ is called an entire function. For meromorphic function $f$, we will use $S(r, f)$ to denote any quantity satisfying $S(r, f)=o(T(r, f))$ for all $r$ outside a possible exceptional set $E$ of finite logarithmic measure $\lim _{r \rightarrow \infty} \int_{[1, r) \cap E} \frac{d t}{t}<$ $\infty$. A meromorphic function $a(z)$ is called small function with respect to $f$ if $T(r, a(z))=S(r, f)$ and the order, hyper order of meromorphic function $f$ are defined by

$$
\rho(f)=\limsup _{r \rightarrow \infty} \frac{\log T(r, f)}{\log r}, \rho_{2}(f)=\limsup _{r \rightarrow \infty} \frac{\log \log T(r, f)}{\log r} .
$$

In addition, for some $a \in \mathbb{C} \cup\{\infty\}$, if the zeros of $f(z)-a$ and $g(z)-a$ (if $a=\infty$, zeros of $f(z)-a$ and $g(z)-a$ are the poles of $f(z)$ and $g(z)$ respectively) coincide in locations and multiplicities we say that $f(z)$ and $g(z)$ share the value $a C M$ (counting multiplicities) and if coincide in locations only we say that $f(z)$ and $g(z)$ share a $I M$ (ignoring multiplicities). 
Definition 1.1 (see $[14,15])$. Let $l$ be a nonnegative integer or infinity. For $a \in \mathbb{C} \cup\{\infty\}$, we denote by $E_{l}(a ; f)$ the set of all a-points of $f$ where an $a$ point of multiplicity $k$ is counted $k$ times if $k \leq l$ and $l+1$ times if $k>l$. If $E_{l}(a ; f)=E_{l}(a ; g)$, we say that $f, g$ share the value a with weight $l$.

Recently, the topic of difference equation and difference product in the complex plane $\mathbb{C}$ has attracted many mathematicians, many papers have focused on value distribution of differences and differences operator analogues of Nevanlinna theory (including $[3,6,8,7,17,23]$ ), and many people dealt with the uniqueness of differences and difference polynomials of meromorphic function and obtained some interesting results $([11,12,20,21,22,27,28])$.

In 2010 and 2011, Zhang [33], Qi [25] studied the problem on the difference polynomials of entire functions sharing small function by using two different methods and obtained the following results.

Theorem 1.1 [33, Theorem 6] or [25, Theorem 2]. Let $f$ and $g$ be transcendental entire functions of finite order, and $\alpha(z)$ be a small function with respect to both $f(z)$ and $g(z)$, let $c$ be a non-zero complex constant, and let $n \geq 7$ be an integer. If $f(z)^{n}(f(z)-1) f(z+c)$ and $g(z)^{n}(g(z)-1) g(z+c)$ share $\alpha(z)$ $C M$, then $f(z) \equiv g(z)$.

Theorem 1.2 ([26, Theorem 1.2]). Let $f$ and $g$ be transcendental entire functions of finite order, and $c$ be a nonzero complex constant, and let $n \geq 6$. If $f^{n} f(z+c)$ and $g^{n} g(z+c)$ share $1 C M$, then $f g=t_{1}$ or $f=t_{2} g$ for some constants $t_{1}$ and $t_{2}$ that satisfies $t_{1}^{n+1}=t_{2}^{n+1}=1$.

In the same year, Li et al. [19] obtained the following theorems which are improvement of Theorem 1.1 and Theorem 1.2.

Theorem 1.3 [19, Theorem 1.1]. Let $f, g$ be transcendental entire functions of finite order, c be a nonzero complex number, $n$ be an integer such that $2 \operatorname{deg} P_{0}<$ $n+1$ and $f(z)^{n} f(z+c)$ and $g(z)^{n} g(z+c)$ share $P_{0}(z) C M$.

(I) If $n \geq 4$ and that $f(z)^{n} f(z+c) /\left(P_{0}(z)\right)$ is a Möbius transformation of $g(z)^{n} g(z+c) /\left(P_{0}(z)\right)$, then either

(i) $f \equiv t g$, where $t \neq 1$ is a constant satisfying $t^{n+1}=1$, or

(ii) $f=e^{Q}, g=t e^{-Q}$, where $P_{0}$ reduces to a nonzero constant $c$, say, and $t$ is a constant such that $t^{n+\lambda}=c^{2}, Q$ is a nonconstant polynomial.

(II) If $n \geq 6$, then (I)(i) or (I)(ii) holds. 
Theorem 1.4 [19, Theorem 1.2]. Let $f$ and $g$ be transcendental entire functions of finite order, and $\alpha(z)$ be a meromorphic function such that $\rho(\alpha)<\rho(f)$, let $c$ be a non-zero complex constant, and let $n \geq 7$ be an integer. If $f(z)^{n}(f(z)-$ 1) $f(z+c)-\alpha(z)$ and $g(z)^{n}(g(z)-1) g(z+c)-\alpha(z)$ share $O C M$, then $f(z) \equiv g(z)$.

In 2012, Chen and Chen [2] further studied the uniqueness of difference polynomials $f^{n}\left(f^{m}-1\right) \prod_{j=1}^{d} f\left(z+c_{j}\right)^{s_{j}}$ and $g^{n}\left(g^{m}-1\right) \prod_{j=1}^{d} g\left(z+c_{j}\right)^{s_{j}}$ sharing small function, where $c_{j} \in \mathbb{C} \backslash\{0\},(j=1, \ldots, d)$ are distinct constants, $n, m, d, s_{j}(j=1, \ldots, d) \in \mathbb{N}_{+}$and obtained the following theorem.

Theorem 1.5 [2, Theorem 1.3]. Let $f$ and $g$ be two transcendental entire functions of finite order, $c_{j} \in \mathbb{C} \backslash\{0\},(j=1, \ldots, d)$ be distinct constants, $n, m$, $d, s_{j}(j=1, \ldots, d) \in \mathbb{N}_{+}$, and $\alpha(z)$ be a small function with respect to both $f(z)$ and $g(z)$. If $n \geq m+8 \lambda$, and $f^{n}\left(f^{m}-1\right) \prod_{j=1}^{d} f\left(z+c_{j}\right)^{s_{j}}$ and $g^{n}\left(g^{m}-\right.$ 1) $\prod_{j=1}^{d} g\left(z+c_{j}\right)^{s_{j}}$ share $\alpha(z) C M$, then $f(z) \equiv \operatorname{tg}(z)$, where $t^{m}=t^{n+\lambda}=1$ and $\lambda=s_{1}+s_{2}+\cdots+s_{d}$.

Let $P(z)=a_{n} z^{n}+a_{n-1} z^{n-1}+\cdots+a_{0}$ be a nonzero polynomial, where $a_{0}, \ldots, a_{n}(\neq 0)$ are complex constants, and $n$ an integer, let $\Gamma_{0}=m_{1}+m_{2}$ and $\Gamma_{1}=m_{1}+2 m_{2}$, where $m_{1}$ is the number of the simple zero of $P(z)$, and $m_{2}$ is the number of multiple zeros of $P(z)$. In 2011, Luo and Lin [24] further investigated the uniqueness of complex difference polynomials of entire functions sharing one value and obtained the following result.

Theorem 1.6 [24, Theorem 2]. Let $f$ and $g$ be transcendental entire functions of finite order, $c$ be a nonzero complex constant, and let $n>2 \Gamma_{1}+1$ be an integer. If $P(f) f(z+c)$ and $P(g) g(z+c)$ share $1 C M$, then one of the following results holds:

(i) $f \equiv$ tg for a constant $t$ such that $t^{l}=1$, where $l=G C D\left\{\lambda_{0}+1, \lambda_{1}+\right.$ $\left.1, \cdots, \lambda_{n}+1\right\}$ and

$$
\lambda_{i}=\left\{\begin{array}{ll}
i, & a_{i} \neq 0, \\
n, & a_{i}=0,
\end{array} \quad i=0,1,2, \ldots, n .\right.
$$

(ii) $f$ and $g$ satisfy the algebraic equation $R(f, g) \equiv 0$, where $R\left(\omega_{1}, \omega_{2}\right)=$ $P\left(\omega_{1}\right) \omega_{1}(z+c)-P\left(\omega_{2}\right) \omega_{2}(z+c) ;$

(iii) $f(z)=e^{\alpha(z)}, g(z)=e^{\beta(z)}$, where $\alpha(z)$ and $\beta(z)$ are two polynomials, $b$ is a constant satisfying $\alpha+\beta \equiv b$ and $a_{n}^{2} e^{(n+1) b}=1$. 
Remark 1.1 The following example shows that the second case of Theorem 1.6 may occur. Let $P(z)=(z-1)^{6}(z+1)^{6} z^{11}, f(z)=\sin z, g(z)=\cos z$ and $c=2 \pi$. It is easy to see that $n>2 \Gamma_{1}+1$ and $P(f) f(z+c) \equiv P(g) g(z+c)$, so $P(f) f(z+c)$ and $P(g) g(z+c)$ share $1 C M$.

Clearly, we get $f \equiv$ tg for a constant $t$ such that $t^{m}=1$, where $m \in Z^{+}$, but $f$ and $g$ satisfy the algebraic equation $R(f, g) \equiv 0$, where $R\left(\omega_{1}, \omega_{2}\right)=$ $P\left(\omega_{1}\right) \omega_{1}(z+c)-P\left(\omega_{2}\right) \omega_{2}(z+c)$.

Regarding this remark, it is a natural question to ask: What condition on $f$ and $g$ can guarantee that the case (ii) of Theorem 1.6 may not occur? The main purpose of this paper is to investigate the above problem; we obtain some theorems which are improvements of Theorems 1.1-1.6.

Let

$$
F(z)=P(f) \prod_{j=1}^{d} f\left(z+c_{j}\right)^{s_{j}}, \quad G(z)=P(g) \prod_{j=1}^{d} g\left(z+c_{j}\right)^{s_{j}} .
$$

Theorem 1.7 Let $f, g$ be transcendental entire functions of finite order such that $f$ and $g$ share $0 C M, F(z), G(z)$ be stated as in (1), where $c_{j} \in \mathbb{C}, n, d, s_{j}(j=$ $1,2, \ldots, d) \in \mathbb{N}_{+}$. If $F(z)$ and $G(z)$ share $1 C M$ and $n>2 \Gamma_{1}+\lambda$, then one of the following cases holds:

(i) $f \equiv$ tg for a constant $t$ such that $t^{\kappa}=1$ where $\kappa=G C D\left\{\lambda_{0}+\lambda, \lambda_{1}+\right.$ $\left.\lambda, \cdots, \lambda_{n}+\lambda\right\}$ and $\lambda_{i}(i=0,1, \ldots, n)$ are stated as in Theorem 1.6;

(ii) $f=e^{\gamma}, g=\zeta e^{-\gamma}$, where $\gamma$ is a nonconstant polynomial, $\zeta$ is a complex constant satisfying $a_{n}^{2} \zeta^{n+\lambda} \equiv 1$.

Remark 1.2 From Theorem 1.6, we see that the condition " $f$ and $g$ share 0 $C M$ " in Theorem 1.7 is necessary.

Theorem 1.8 Under the assumptions of Theorem 1.7, if

$$
E_{k}(1 ; F(z))=E_{k}(1 ; G(z))
$$

and $k, n, d(>0), s_{j}(>0)(j=1, \ldots, d)$ are integers satisfying one of the following conditions:
(I) $k \geq 3, n>2 \Gamma_{1}+\lambda$;
(II) $k=2, n>2 \Gamma_{1}+\Gamma_{0}+\lambda+d$;
(III) $k=1, n>2 \Gamma_{1}+2 \Gamma_{0}+\lambda+2 d$;
(IV) $k=0, n>2 \Gamma_{1}+3 \Gamma_{0}+\lambda+3 d$. 
Then the conclusions of Theorem 1.7 hold.

Let

$$
F_{1}(z)=\frac{P(f) \prod_{j=1}^{d} f\left(z+c_{j}\right)^{s_{j}}}{P_{0}(z)}, \quad G_{1}(z)=\frac{P(g) \prod_{j=1}^{d} g\left(z+c_{j}\right)^{s_{j}}}{P_{0}(z)} .
$$

Theorem 1.9 Let $f, g$ be transcendental entire functions of finite order such that $f$ and $g$ share $O C M, F_{1}(z), G_{1}(z)$ be stated as in (2), let $n$ be an integer such that $\operatorname{deg} P_{0}<n+\lambda$ and $F_{1}(z)$ and $G_{1}(z)$ share $P_{0}(z) C M$. If $n>2\left(\Gamma_{0}+d\right)-\lambda$ and that $F_{1}$ is a Möbius transformation of $G_{1}$, or if $n>3 \Gamma_{0}+2 \Gamma_{1}+\lambda+3 d$, then one of the following cases holds:

(i) $f \equiv$ tg for a constant $t$ such that $t^{\kappa}=1$ where $\kappa=G C D\left\{\lambda_{0}+\lambda, \lambda_{1}+\right.$ $\left.\lambda, \cdots, \lambda_{n}+\lambda\right\}$ and $\lambda_{i}(i=0,1, \ldots, n)$ is stated as in Theorem 1.6;

(ii) $f=e^{\gamma}, g=\zeta e^{-\gamma}$, where $P_{0}$ reduces to a nonzero constant c, say, and $\zeta$ is a constant such that $a_{n}^{2} \zeta^{n+\lambda}=c^{2}$, $\gamma$ is a nonconstant polynomial.

Theorem 1.10 Under the assumptions of Theorem 1.9, if

$$
E_{k}\left(1 ; F_{1}(z)\right)=E_{k}\left(1 ; G_{1}(z)\right) .
$$

If $n>2\left(\Gamma_{0}+d\right)-\lambda$ and that $F_{1}$ is a Möbius transformation of $G_{1}$, or if $k, n, d(>0), s_{j}(>0)(j=1, \ldots, d)$ are integers satisfying one of the following conditions:

(I) $k \geq 3, n>2 \Gamma_{1}+\lambda$;

(II) $k=2, n>2 \Gamma_{1}+\Gamma_{0}+\lambda+d ;$

(III) $k=1, n>2 \Gamma_{1}+2 \Gamma_{0}+\lambda+2 d$;

(IV) $k=0, n>2 \Gamma_{1}+3 \Gamma_{0}+\lambda+3 d$.

Then the conclusions of Theorem 1.9 hold.

From Theorem 1.9 and Theorem 1.10, one can get the following corollaries immediately.

Corollary 1.1 Let $f, g$ be transcendental entire functions of finite order such that $f$ and $g$ share $0 C M, F_{1}(z), G_{1}(z)$ be stated as in (2), and $F_{1}(z)$ and $G_{1}(z)$ share $z C M$. If $n>2\left(\Gamma_{0}+d\right)-\lambda$ and that $F_{1}$ is a Möbius transformation of $G_{1}$, or if $n>3 \Gamma_{0}+2 \Gamma_{1}+\lambda+3 d$, then $f \equiv$ tg for a constant $t$ such that $t^{\kappa}=1$ where $\kappa=G C D\left\{\lambda_{0}+\lambda, \lambda_{1}+\lambda, \cdots, \lambda_{n}+\lambda\right\}$ and $\lambda_{i}(i=0,1, \ldots, n)$ is stated as in Theorem 1.6. 
Corollary 1.2 Under the assumptions of Theorem 1.9, if

$$
E_{k}\left(z ; F_{1}(z)\right)=E_{k}\left(z ; G_{1}(z)\right) .
$$

If $n>2\left(\Gamma_{0}+d\right)-\lambda$ and that $F_{1}$ is a Möbius transformation of $G_{1}$, or if $k, n, d(>0), s_{j}(>0)(j=1, \ldots, d)$ are integers satisfying one of the following conditions:

(I) $k \geq 3, n>2 \Gamma_{1}+\lambda$;

(II) $k=2, n>2 \Gamma_{1}+\Gamma_{0}+\lambda+d ;$

(III) $k=1, n>2 \Gamma_{1}+2 \Gamma_{0}+\lambda+2 d$;

(IV) $k=0, n>2 \Gamma_{1}+3 \Gamma_{0}+\lambda+3 d$.

Then $f \equiv$ tg for a constant $t$ such that $t^{\kappa}=1$ where $\kappa=G C D\left\{\lambda_{0}+\lambda, \lambda_{1}+\right.$ $\left.\lambda, \cdots, \lambda_{n}+\lambda\right\}$ and $\lambda_{i}(i=0,1, \ldots, n)$ is stated as in Theorem 1.5.

Let

$$
\begin{aligned}
& F_{2}(z)=P(f) \prod_{j=1}^{d} f\left(z+c_{j}\right)^{s_{j}}-\alpha(z), \\
& G_{2}(z)=P(g) \prod_{j=1}^{d} g\left(z+c_{j}\right)^{s_{j}}-\alpha(z),
\end{aligned}
$$

where $\alpha(z)$ be a small function with respect to both $f(z)$ and $g(z)$.

Theorem 1.11 Let $f, g$ be transcendental entire functions of finite non-integer order such that $f$ and $g$ share $0 C M, F_{2}(z), G_{2}(z)$ be stated as in (3), and $F_{2}(z)$ and $G_{2}(z)$ share $\alpha(z) C M$. If $n>2 \Gamma_{0}+\lambda$, then $f \equiv$ tg for a constant $t$ such that $t^{\kappa}=1$ where $\kappa=G C D\left\{\lambda_{0}+\lambda, \lambda_{1}+\lambda, \cdots, \lambda_{n}+\lambda\right\}$ and $\lambda_{i}(i=$ $0,1, \ldots, n)$ is stated as in Theorem 1.6.

Theorem 1.12 Under the assumptions of Theorem 1.11, if

$$
E_{k}\left(1 ; F_{2}(z)\right)=E_{k}\left(1 ; G_{2}(z)\right)
$$

and $k, n, d(>0), s_{j}(>0)(j=1, \ldots, d)$ are integers satisfying one of the following conditions:

$$
\text { (I) } k \geq 3, n>2 \Gamma_{1}+\lambda \text {; }
$$


(II) $k=2, n>2 \Gamma_{1}+\Gamma_{0}+\lambda+d ;$

(III) $k=1, n>2 \Gamma_{1}+2 \Gamma_{0}+\lambda+2 d$;

(IV) $k=0, n>2 \Gamma_{1}+3 \Gamma_{0}+\lambda+3 d$.

Then $f \equiv$ tg for a constant $t$ such that $t^{\kappa}=1$ where $\kappa=G C D\left\{\lambda_{0}+\lambda, \lambda_{1}+\right.$ $\left.\lambda, \cdots, \lambda_{n}+\lambda\right\}$ and $\lambda_{i}(i=0,1, \ldots, n)$ is stated as in Theorem 1.6.

Remark 1.3 The following example shows that Theorem 1.11 and Theorem 1.12 may not hold for entire functions of finite integer order.

Example 1.1 Let $P(z)=z^{4}, f(z)=(z+2)^{2}(z+3) e^{(z-2)^{2}}, g(z)=(z+$ $2)^{2}(z+3) e^{-(z-2)^{2}}, \alpha(z)=(z+2)^{8}(z+3)^{4}(z+4)^{2}(z+5), d=1, s_{1}=1$ and $c_{1}=2$. Thus, we have that $f, g$ are of finite integer order 2 and $f, g$ share 0 $C M, \Gamma_{0}=1, \lambda=1$ and $n=4>3=2 \Gamma_{0}+\lambda$, moreover, $P(f) f(z+c)$ and $P(g) g(z+c)$ share $\alpha(z) C M$. But, we get $f \not \equiv t g$ for a constant $m$ such that $t^{m}=1$, where $m \in \mathbb{Z}^{+}$.

\section{Some lemmas}

To prove our theorems, we require some lemmas as follows.

Lemma 2.1 [29]. Let $f$ be a nonconstant meromorphic function and $P(f)=$ $a_{0}+a_{1} f+a_{2} f^{2}+\cdots+a_{n} f^{n}$, where $a_{0}, a_{1}, a_{2}, \cdots, a_{n}$ are constants and $a_{n} \neq 0$. Then

$$
T(r, P(f))=n T(r, f)+S(r, f)
$$

Lemma 2.2 [9, Theorem 5.1]. Let $f$ be a transcendental meromorphic function of $\rho_{2}(f)<1, \varsigma<1$, $\varepsilon$ be an enough small number. Then

$$
m\left(r, \frac{f(z+c)}{f(z)}\right)=o\left(\frac{T(r, f)}{r^{1-\varsigma-\varepsilon}}\right)=S(r, f),
$$

for all $r$ outside of a set of finite logarithmic measure.

Lemma 2.3 [9, Lemma 8.3]. Let $T:[0,+\infty) \rightarrow[0,+\infty)$ be a non-decreasing continuous function and let $s \in(0,+\infty)$. If the hyper order of $T$ is strictly less that one, that is,

$$
\limsup _{r \rightarrow \infty} \frac{\log \log T(r)}{\log r}<1
$$


and $\delta \in(0,1-\varsigma)$, then

$$
T(r+s)=T(r)+o\left(\frac{T(r)}{r^{\delta}}\right)
$$

for all r runs to infinity outside of a set of finite logarithmic measure.

Lemma 2.4 [13, Lemma 2.2]. Let $\varphi(r)$ be a nondecreasing, continuous function on $\mathbb{R}_{+}$. Suppose that

$$
0<\rho<\limsup _{r \rightarrow+\infty} \frac{\log \varphi(r)}{\log r}
$$

and set

$$
I=\left\{t: t \in R_{+}, \varphi(r) \geq r^{\rho}\right\}
$$

Then we have

$$
\overline{\log d e n s} I=\limsup _{r \rightarrow+\infty} \frac{\int_{I \cap[1, r]} \frac{d r}{r}}{\log r}>0 .
$$

Lemma 2.5 [31, Lemma 7.1]. Let $f, g$ be two nonconstant meromorphic functions such that $g$ is a Möbius transformation of $f$. Suppose that there exists a subset $I \subset R_{+}$with its linear measure mesI $=+\infty$ such that

$$
\bar{N}\left(r, \frac{1}{f}\right)+\bar{N}(r, f)+\bar{N}\left(r, \frac{1}{g}\right)+\bar{N}(r, g)<(\lambda+o(1)) T(r, f),
$$

as $r \in I$ and $r \rightarrow+\infty$, where $\lambda<1$. If there exists a point $z_{0} \in \mathbb{C}$ such that $f\left(z_{0}\right)=g\left(z_{0}\right)=1$, then $f=g$ or $f g=1$.

In the following, we explain some definitions and notations which are used in this paper. For $a \in \mathbb{C} \cup \infty$ and $k$ is a positive integer, we denote by $\bar{N}_{(k}\left(r, \frac{1}{f-a}\right)$ the counting function of those $a$-points of $f$ whose multiplicities are not less than $k$ in counting the $a$-points of $f$ we ignore the multiplicities (see $[10,30]$ ) and

$$
N_{k}\left(r, \frac{1}{f-a}\right)=\bar{N}\left(r, \frac{1}{f-a}\right)+\bar{N}_{(2}\left(r, \frac{1}{f-a}\right)+\cdots+\bar{N}_{(k}\left(r, \frac{1}{f-a}\right) .
$$

Definition 2.1 [1]. When $f$ and $g$ share $1 I M$, we denote by $\bar{N}_{L}\left(r, \frac{1}{f-1}\right)$ the counting function of the 1-points of $f$ whose multiplicities are greater than 1points of $g$, where each zero is counted only once; similarly, we have $\bar{N}_{L}\left(r, \frac{1}{g-1}\right)$. Let $z_{0}$ be a zero of $f-1$ of multiplicity $p$ and a zero of $g-1$ of multiplicity $q$, we also denote by $N_{11}\left(r, \frac{1}{f-1}\right)$ the counting function of those 1-points of $f$ where $p=q=1$. 
Lemma 2.6 [31, Theorem 7.2] Let $f, g$ be two nonconstant meromorphic functions such that $f, g$ share $1, \infty C M$. Suppose that there exists a subset $I \subset \mathbb{R}_{+}$ with its linear measure mesI $=+\infty$ such that

$$
N_{2}\left(r, \frac{1}{f}\right)+N_{2}\left(r, \frac{1}{g}\right)+2 \bar{N}(r, f)<\mu T(r)+S(r),
$$

as $r \in I$ and $r \rightarrow+\infty$, where $\mu<1, T(r)=\max \{T(r, f), T(r, g)\}$ and $S(r)=o(T(r))$, as $r \in I$ and $r \rightarrow+\infty$. Then $f=g$ or $f g=1$.

Lemma 2.7 [5]. Let $f$ and $g$ be two meromorphic functions. If $f$ and $g$ share 1 $C M$, then one of the following three cases holds:

(i)

$$
\begin{aligned}
T(r, f)+T(r, g) \leq & 2 N_{2}(r, f)+2 N_{2}(r, g)+2 N_{2}\left(r, \frac{1}{f}\right)+2 N_{2}\left(r, \frac{1}{g}\right) \\
& +S(r, f)+S(r, g)
\end{aligned}
$$

(ii) $f \equiv g$;

(iii) $f \cdot g=1$.

Lemma 2.8 [4, Lemma 2]. Let $f$ and $g$ be two meromorphic functions, and let $k$ be a positive integer. If $E_{k}(1 ; f)=E_{k}(1 ; g)$, then one of the following cases must occur:

(i)

$$
\begin{aligned}
& T(r, f)+T(r, g) \leq N_{2}(r, f)+N_{2}(r, g)+N_{2}\left(r, \frac{1}{f}\right)+N_{2}\left(r, \frac{1}{g}\right) \\
& \quad+\bar{N}\left(r, \frac{1}{f-1}\right)+\bar{N}\left(r, \frac{1}{g-1}\right)-N_{11}\left(r, \frac{1}{f-1}\right)+\bar{N}_{(k+1}\left(r, \frac{1}{f-1}\right) \\
& \quad+\bar{N}_{(k+1}\left(r, \frac{1}{g-1}\right)+S(r, f)+S(r, g)
\end{aligned}
$$

(ii) $f=\frac{(b+1) g+(a-b-1)}{b g+(a-b)}$, where $a(\neq 0)$, b are two constants.

Lemma 2.9 [32, Lemma 4 and proof of Theorem 1]. Let $f$ and $g$ be two entire functions. If $f$ and $g$ share 1 IM, then one of the following cases must occur: 
(i)

$$
\begin{aligned}
T(r, f) \leq & N_{2}\left(r, \frac{1}{f}\right)+N_{2}\left(r, \frac{1}{g}\right)+2 \bar{N}_{L}\left(r, \frac{1}{f-1}\right)+\bar{N}_{L}\left(r, \frac{1}{g-1}\right) \\
& +S(r, f)+S(r, g),
\end{aligned}
$$

the same inequality holding for $T(r, g)$;

(ii) $f=g$;

(iii) $f \cdot g=1$.

Remark 2.1 Let $f$ and $g$ be two nonconstant meromorphic functions, for a $\in$ $C \cup\{\infty\}$, and let $\bar{N}_{E}(r, a)$ count those points in $\bar{N}\left(r, \frac{1}{f-a}\right)$, where a is taken by $f$ and $g$ with the same multiplicity, and each point is counted only once, and $\bar{N}_{0}(r, a)$ as ignoring multiplicities, $\bar{N}\left(r, \frac{1}{f-a}\right)=\bar{N}(r, f)$ as $a=\infty$. We say that $f, g$ share the value a $C M *$, if

$$
\bar{N}\left(r, \frac{1}{f-a}\right)-\bar{N}_{E}(r, a)=S(r, f), \bar{N}\left(r, \frac{1}{g-a}\right)-\bar{N}_{E}(r, a)=S(r, g),
$$

and $f, g$ share the value a $I M^{*}$, if $\bar{N}_{E}(r, a)$ is replaced by $\bar{N}_{0}(r, a)$.

Thus, from the proof of Theorem 1 in ref.[32], Theorem 1.48 and Theorem 7.10 in ref. [31], we can get that Lemmas 2.6-2.9 still hold if $C M(I M)$ is replaced by $C M^{*}\left(I M^{*}\right)$.

Lemma 2.10 [18, Lemma 3]. Suppose that h is a nonconstant meromorphic function satisfying

$$
\bar{N}(r, h)+\bar{N}\left(r, \frac{1}{h}\right)=S(r, h) .
$$

Let $f=a_{0} h^{p}+a_{1} h^{p-1}+\cdots+a_{p}$ and $g=b_{0} h^{q}+b_{1} h^{q-1}+\cdots+b_{q}$ be polynomials in $h$ with coefficients $a_{0}, a_{1}, \ldots, a_{p}, b_{0}, b_{1}, \ldots, b_{q}$ being small functions of $h$ and $a_{0} b_{0} a_{p} \not \equiv 0$. If $q \leq p$, then $m\left(r, \frac{g}{f}\right)=S(r, h)$.

Lemma 2.11 Let $f(z)$ be a nonconstant meromorphic function of finite order $\rho(f)<+\infty, c_{j} \in \mathbb{C} \backslash\{0\},(j=1, \ldots, d)$ be distinct constants, $P(z)=$ $a_{n} z^{n}+a_{n-1} z^{n-1}+\cdots+a_{0}$ be a nonzero polynomial, where $a_{0}, \ldots, a_{n}(\neq 0)$ are complex constants, and $n$ an integer. Let $F(z)$ be stated as (1), then

$$
m(r, F(z))=(n+\lambda) m(r, f(z))+o\left(\frac{T(r, f(z))}{r^{1-\varepsilon}}\right)+O(1),
$$

for all $r$ outside of a set of finite logarithmic measure, where $\lambda=s_{1}+s_{2}+\cdots+s_{d}$. 
Proof: Since $f$ is a nonconstant meromorphic function of finite order, then $\rho_{2}(f)=0<1$. Thus, by Lemma 2.2, the assumptions of Lemma 2.11, the proof of Theorem 1.12 in [31] and the standard Valiron-Mokhon'ko lemma, we have

$$
\begin{aligned}
(n+\lambda) m(r, f(z)) & =m\left(r, f(z)^{\lambda} P(f)\right)+O(1) \\
& \leq m\left(r, \frac{f(z)^{\lambda} P(f)}{F(z)}\right)+m(r, F(z))+O(1) \\
& \leq \sum_{j=1}^{d} s_{j} m\left(r, \frac{f(z)}{f\left(z+c_{j}\right)}\right)+m(r, F(z))+O(1) \\
& \leq m(r, F(z))+o\left(\frac{T(r, f(z))}{r^{1-\varepsilon}}\right)+O(1)
\end{aligned}
$$

that is,

$$
m(r, F(z)) \geq(n+\lambda) m(r, f(z))+o\left(\frac{T(r, f(z))}{r^{1-\varepsilon}}\right)+O(1) .
$$

On the other hand, from Lemma 2.2, the proof of Theorem 1.12 in [31] and the standard Valiron-Mokhon'ko lemma, we get

$$
\begin{aligned}
m(r, F(z)) & \leq m(r, P(f))+m\left(r, f(z)^{\lambda} \prod_{j=1}^{d} \frac{f\left(z+c_{j}\right)^{s_{j}}}{f(z)^{s_{j}}}\right)+O(1) \\
& \leq n m(r, f(z))+\lambda m(r, f(z))+\sum_{j=1}^{d} s_{j} m\left(r, \frac{f\left(z+c_{j}\right)}{f(z)}\right)+O(1) \\
& \leq(n+\lambda) m(r, f(z))+o\left(\frac{T(r, f(z))}{r^{1-\varepsilon}}\right)+O(1)
\end{aligned}
$$

From (4) and (5), we can prove this lemma easily.

Lemma 2.12 Let $f(z)$ and $g(z)$ be transcendental entire functions of finite orders, $c_{j} \in \mathbb{C} \backslash\{0\},(j=1, \ldots, d)$ be distinct constants, $n, d, s_{j}(j=1, \ldots, d) \in$ $\mathbb{N}_{+}$, and $\alpha(z)$ be a small function of $f$ and $g, P(z)$ be stated as in Lemma 2.12, and let $n$ be an integer such that $n>\Gamma_{1}$. If $P(f) \prod_{j=1}^{d} f\left(z+c_{j}\right)^{s_{j}}$ and $P(g) \prod_{j=1}^{d} g\left(z+c_{j}\right)^{s_{j}}$ share $\alpha(z) I M$, then $\rho(f)=\rho(g)$. 
Proof: Let

$$
F^{*}(z)=\frac{P(f) \prod_{j=1}^{d} f\left(z+c_{j}\right)^{s_{j}}}{\alpha(z)}, \quad G^{*}(z)=\frac{P(g) \prod_{j=1}^{d} g\left(z+c_{j}\right)^{s_{j}}}{\alpha(z)},
$$

then from the assumptions of Lemma 2.12, and by Lemma 2.11, we have $F^{*}(z)$ and $G^{*}(z)$ share $1 I M$ and

$$
T\left(r, F^{*}(z)\right)=(n+\lambda) T(r, f(z))+o\left(\frac{T(r, f(z))}{r^{1-\varepsilon}}\right)+O(\log r)
$$

and

$$
T\left(r, G^{*}(z)\right)=(n+\lambda) T(r, g(z))+o\left(\frac{T(r, g(z))}{r^{1-\varepsilon}}\right)+O(\log r)
$$

as $r \rightarrow \infty$ and $r \notin E$, where and in what follows, $E \subset[0,+\infty)$ is a set with its logarithmic measure $\log$ mes $E<\infty$. Since $f$ is of finite order $\rho(f)<+\infty$, then $\rho_{2}(f)=0<1$. Thus, if follows from Lemma 2.3 that

$$
\begin{aligned}
\bar{N}\left(r, \frac{1}{f\left(z+c_{j}\right)}\right) & \leq \bar{N}\left(r+\left|c_{j}\right|, \frac{1}{f(z)}\right) \leq T\left(r+\left|c_{j}\right|, f(z)\right)+O(1) \\
& =T(r, f(z))+o\left(\frac{T(r, f(z))}{r^{\delta}}\right)+O(1)
\end{aligned}
$$

as $r \rightarrow \infty$ and $r \notin E$, where and in what follows, $\delta \in(0,1)$ is a positive real number. So, by using the second fundamental theorem, we have

$$
\begin{aligned}
T\left(r, F^{*}(z)\right) \leq & \bar{N}\left(r, F^{*}(z)\right)+\bar{N}\left(r, \frac{1}{F^{*}(z)}\right)+\bar{N}\left(r, \frac{1}{F^{*}(z)-1}\right)+O(\log r) \\
\leq & \bar{N}\left(r, \frac{1}{P(f)}\right)+\sum_{j=1}^{d} \bar{N}\left(r, \frac{1}{f\left(z+c_{j}\right)}\right)+\bar{N}\left(r, \frac{1}{G^{*}(z)-1}\right) \\
& +O(\log r) \\
\leq & \left(\Gamma_{1}+d\right) T(r, f(z))+T\left(r, G^{*}(z)\right)+o\left(\frac{T(r, f(z))}{r^{\delta}}\right) \\
& +O(\log r)
\end{aligned}
$$

as $r \rightarrow \infty$ and $r \notin E$. Then combining (6) and (7), we have

$$
\begin{aligned}
(n+\lambda) T(r, f(z)) \leq & \left(\Gamma_{1}+d\right) T(r, f(z))+(n+\lambda) T(r, g(z))+o\left(\frac{T(r, f(z))}{r^{\delta}}\right) \\
& +o\left(\frac{T(r, g(z))}{r^{\delta}}\right)+O(\log r) .
\end{aligned}
$$


Since $n>\Gamma_{1}+d-\lambda$, it follows by Lemma 1.1.2 in [16] that

$$
\rho(f) \leq \rho(g)
$$

Similarly, we have

$$
\rho(g) \leq \rho(f) .
$$

Thus, it follows from (8) and (9) that $\rho(g)=\rho(f)$.

\section{Proofs of theorems 1.7 and 1.8}

\subsection{The proof of theorem 1.7}

Since $f, g$ are entire functions of finite order, from the form of $F(z)$, we have by Lemmas 2.1-2.3 that

$$
\begin{aligned}
& N_{2}\left(r, \frac{1}{F(z)}\right) \leq N_{2}\left(r, \frac{1}{P(f)}\right)+N_{2}\left(r, \frac{1}{\prod_{j=1}^{d} f\left(z+c_{j}\right)}\right)+O(1) \\
& \leq \Gamma_{1} T(r, f(z))+\sum_{j=1}^{d} s_{j} T\left(r, f\left(z+c_{j}\right)\right)+O(1) \\
& \quad \leq\left(\Gamma_{1}+\lambda\right) T(r, f(z))+o\left(\frac{T(r, f(z))}{r^{\delta}}\right)+o\left(\frac{T(r, f(z))}{r^{1-\varepsilon}}\right)+O(1) .
\end{aligned}
$$

Thus, it follows from (6) that

$$
\begin{aligned}
N_{2}\left(r, \frac{1}{F(z)}\right) & \leq \frac{\Gamma_{1}+\lambda}{n+\lambda} T(r, F(z))+o\left(\frac{T(r, f(z))}{r^{\delta}}\right)+o\left(\frac{T(r, f(z))}{r^{1-\varepsilon}}\right)+O(1) \\
& \leq\left(\frac{\Gamma_{1}+\lambda}{n+\lambda}+o(1)\right) T(r, F(z))
\end{aligned}
$$

as $r \rightarrow+\infty$ and $r \notin E$. Similarly,

$$
\begin{aligned}
N_{2}\left(r, \frac{1}{G(z)}\right) & \leq \frac{\Gamma_{1}+\lambda}{n+\lambda} T(r, G(z))+o\left(\frac{T(r, g(z))}{r^{\delta}}\right)+o\left(\frac{T(r, g(z))}{r^{1-\varepsilon}}\right)+O(1) \\
& \leq\left(\frac{\Gamma_{1}+\lambda}{n+\lambda}+o(1)\right) T(r, G(z))
\end{aligned}
$$

as $r \rightarrow+\infty$ and $r \notin E$. From (10), (11) and $f, g$ are entire functions, it follows

$$
N_{2}\left(r, \frac{1}{F}\right)+N_{2}\left(r, \frac{1}{G}\right)+2 \bar{N}(r, F) \leq\left(\frac{2 \Gamma_{1}+2 \lambda}{n+\lambda}+o(1)\right) T(r),
$$


as $r \rightarrow+\infty$ and $r \notin E$, where $T(r)=\max \{T(r, F), T(r, G)\}$. Since $n>$ $2 \Gamma_{1}+\lambda$, by Lemma 2.6, we have $F G=1$ or $F=G$. Then, the following two cases will be considered.

Case 1. If $F G=1$, that is,

$$
P(f(z)) \prod_{j=1}^{d} f\left(z+c_{j}\right)^{s_{j}} P(g(z)) \prod_{j=1}^{d} g\left(z+c_{j}\right)^{s_{j}}=1 .
$$

Suppose that the roots of $P(z)=0$ are $b_{1}, b_{2}, \ldots, b_{m}$ with multiplicities $l_{1}, l_{2}, \ldots, l_{m}$. Then we have $l_{1}+l_{2}+\cdots+l_{m}=n$. From (13), we have

$$
\begin{gathered}
\left(f-b_{1}\right)^{l_{1}}\left(f-b_{2}\right)^{l_{2}} \cdots\left(f-b_{m}\right)^{l_{m}} \prod_{j=1}^{d} f\left(z+c_{j}\right)^{s_{j}} \times \\
\left(g-b_{1}\right)^{l_{1}}\left(g-b_{2}\right)^{l_{2}} \cdots\left(g-b_{m}\right)^{l_{m}} \prod_{j=1}^{d} g\left(z+c_{j}\right)^{s_{j}} \equiv 1 .
\end{gathered}
$$

Since $f, g$ are nonconstant entire functions, from (14), we can deduce that $b_{1}=$ $b_{2}=\cdots=b_{m}=0$. If fact, from (14), we can get that $b_{1}, b_{2}, \ldots, b_{m}$ are the Picard exceptional values. If $m \geq 2$ and $b_{j} \neq 0(j=1,2, \ldots, m)$, by Picard's theorem of entire function, we can get that the Picard's exceptional values of $f$ as least three. Thus, we can get a contradiction. Hence, $m=1$ and $l_{1}=n$, that is, there exists a complex constant $a$ satisfying $P(f)=a_{n}(f-a)^{n}$ and $P(g)=a_{n}(g-a)^{n}$. Then

$$
a_{n}(f-a)^{n} \prod_{j=1}^{d} f\left(z+c_{j}\right)^{s_{j}} a_{n}(g-a)^{n} \prod_{j=1}^{d} g\left(z+c_{j}\right)^{s_{j}} \equiv 1 .
$$

Since $f, g$ are transcendental entire functions, by the Picard's theorem, we can get that $f-a=0$ and $g-a=0$ do not have zeros. Then, we obtain that $f(z)=$ $e^{\gamma(z)}+a, g(z)=e^{\beta(z)}+a$ where $\gamma(z), \beta(z)$ are two nonconstant polynomials. We also see that $f\left(z+c_{j}\right) \neq 0$ and $g\left(z+c_{j}\right) \neq 0$ for $j=1,2, \ldots, d$ by (13). Thus, it follows $a=0$, that is, $f(z)=e^{\gamma(z)}, g(z)=e^{\beta(z)}$ and $P(z)=a_{n} z^{n}$. So, from (13), we have

$$
a_{n}^{2} \exp \left\{n[\gamma(z)+\beta(z)]+\sum_{j=1}^{d} s_{j}\left[\gamma\left(z+c_{j}\right)+\beta\left(z+c_{j}\right)\right]\right\} \equiv 1 .
$$

Since $\gamma(z), \beta(z)$ are two nonconstant polynomials, we get $\gamma(z)+\beta(z) \equiv \xi$, where $\xi$ is a constant. Hence, we can easily get that $f(z)=e^{\gamma}$ and $g(z)=$ 
$\zeta e^{-\gamma}$, where $\gamma$ is a nonconstant polynomial, $\zeta$ is a complex constant satisfying $a_{n}^{2} \zeta^{n+\lambda} \equiv 1$ and $\zeta=e^{\xi}$.

Case 2. If $F=G$, that is,

$$
P(f(z)) \prod_{j=1}^{d} f\left(z+c_{j}\right)^{s_{j}}=P(g(z)) \prod_{j=1}^{d} g\left(z+c_{j}\right)^{s_{j}} .
$$

Set $h=\frac{f}{g}$, substituting $f=g h$ into (16), we deduce that

$$
\begin{gathered}
{\left[a_{n} g(z)^{n} h(z)^{n}+a_{n-1} g(z)^{n-1} h(z)^{n-1}+\cdots+a_{0}\right] \prod_{j=1}^{d} h\left(z+c_{j}\right)^{s_{j}}} \\
=a_{n} g(z)^{n}+\cdots+a_{0}
\end{gathered}
$$

where $a_{n}(\neq 0), a_{n-1}, \ldots, a_{0}$ are complex constants. Thus, we get

$$
\begin{aligned}
a_{n} g(z)^{n}\left[h(z)^{n}\right. & \left.\prod_{j=1}^{d} h\left(z+c_{j}\right)^{s_{j}}-1\right]+a_{n-1} g(z)^{n-1}\left[h(z)^{n-1} \times\right. \\
& \left.\prod_{j=1}^{d} h\left(z+c_{j}\right)^{s_{j}}-1\right]+\cdots+a_{0}\left[\prod_{j=1}^{d} h\left(z+c_{j}\right)^{s_{j}}-1\right]=0 .
\end{aligned}
$$

Now, we will consider the following two subcases.

Subcase 2.1. Suppose that $h$ is a constant. Thus, it follows from (17) that

$$
a_{n} g(z)^{n}\left(h^{n+\lambda}-1\right)+a_{n-1} g(z)^{n-1}\left(h^{n-1+\lambda}-1\right)+\cdots+a_{0}\left(h^{n}-1\right)=0 .
$$

We claim $h^{\kappa}=1$, where $\kappa$ is stated as in Theorem 1.7.

In fact, if $a_{n} \neq 0$ and $a_{i}=0$ for $i=0,1, \ldots, n-1$, since $g$ is a transcendental entire function, thus we have $h^{n+\lambda}=1$.

If $a_{n}$ is not the only nonzero coefficient, assume that $h^{n+\lambda} \neq 1$. Then, we can deduce $T(r, g)=S(r, g)$ by (18), a contradiction. Hence $h^{n+\lambda}=1$. By using the similar discussion, we can get that $h^{k+\lambda}=1$ when $a_{k} \neq 0$ for some $k=0,1, \ldots, n$. Thus, we have $f=t g$ for a constant $t$ such that $t^{\kappa}=1$, where $\kappa=G C D\left\{\lambda_{0}+\lambda, \lambda_{1}+\lambda, \cdots, \lambda_{n}+\lambda\right\}$ and $\lambda_{i}(i=0,1, \ldots, n)$ is stated as in Theorem 1.6.

Subcase 2.2. Suppose that $h$ is not a constant. Then we claim

$$
\prod_{j=1}^{d} h\left(z+c_{j}\right)^{s_{j}} h(z)^{n} \equiv 1
$$


In fact, if $a_{n} \neq 0$ and $a_{i}=0$ for $i=0,1, \ldots, n-1$, since $g$ is a transcendental entire function, and from (17), then we have $\prod_{j=1}^{d} h\left(z+c_{j}\right)^{s_{j}} h(z)^{n} \equiv 1$.

If $a_{n}$ is not the only nonzero coefficient, assume that $\prod_{j=1}^{d} h\left(z+c_{j}\right)^{s_{j}} h(z)^{n}$ $\not \equiv 1$. From (17), we have

$$
\begin{gathered}
-a_{n} g(z)^{n}=a_{n-1} g(z)^{n-1} \frac{h(z)^{n-1} \prod_{j=1}^{d} h\left(z+c_{j}\right)^{s_{j}}-1}{h(z)^{n} \prod_{j=1}^{d} h\left(z+c_{j}\right)^{s_{j}}-1} \\
+\cdots+a_{0} \frac{\prod_{j=1}^{d} h\left(z+c_{j}\right)^{s_{j}}-1}{h(z)^{n} \prod_{j=1}^{d} h\left(z+c_{j}\right)^{s_{j}}-1} .
\end{gathered}
$$

Let

$$
H_{i}=\frac{h(z)^{n-i} \prod_{j=1}^{d} h\left(z+c_{j}\right)^{s_{j}}-1}{h(z)^{n} \prod_{j=1}^{d} h\left(z+c_{j}\right)^{s_{j}}-1}, i=1,2, \ldots, n
$$

then we have

$$
H_{i}=\frac{h(z)^{n-i+\lambda} \prod_{j=1}^{d}\left(\frac{h\left(z+c_{j}\right)}{h(z)}\right)^{s_{j}}-1}{h(z)^{n+\lambda} \prod_{j=1}^{d}\left(\frac{h\left(z+c_{j}\right)}{h(z)}\right)^{s_{j}}-1}, i=1,2, \ldots, n .
$$

Since $f, g$ are entire functions and $f, g$ share $0 C M$, then $h$ is an entire function, from Lemma 2.2 and Lemma 2.10, we have $m\left(r, H_{i}\right)=S(r, h)$ for $i=$ $1,2, \ldots, n$. Since $g$ is an entire function and $a_{n} \neq 0$, we can deduce from (20) that $T(r, g)=m(r, g)=S(r, g)$, a contradiction. Then (19) holds.

From the assumptions of Theorem 1.8, we get that $h$ has no zeros and is of finite order, then we can write $h=e^{\omega(z)}$, where $\omega(z)$ is a polynomial. Thus, it follows from (19) that

$$
\exp \left\{\sum_{j=1}^{d} s_{j} \omega\left(z+c_{j}\right)+n \omega(z)\right\} \equiv 1 .
$$


Differentiating the above equation, we have

$$
\sum_{j=1}^{d} s_{j} \omega^{\prime}\left(z+c_{j}\right)+n \omega^{\prime}(z) \equiv 0
$$

Let $\omega(z)=d_{m} z^{m}+d_{m-1} z^{m-1}+\cdots+d_{0}$, where $d_{m} \neq 0$, then

$$
\begin{aligned}
\omega\left(z+c_{j}\right) & =d_{m}\left(z+c_{j}\right)^{m}+d_{m-1}\left(z+c_{j}\right)^{m-1}+\cdots+d_{0} \\
& =d_{m} z^{m}+\left(m d_{m} c_{j}+d_{m-1}\right) z^{m-1}+\cdots \\
\omega^{\prime}(z) & =m d_{m} z^{m-1}+(m-1) d_{m-1} z^{m-2}+\cdots+d_{1} \\
\omega^{\prime}\left(z+c_{j}\right) & =m d_{m} z^{m-1}+(m-1)\left(m d_{m} c_{j}+d_{m-1}\right) z^{m-2}+\cdots \\
\text { for } j=1,2, \ldots, d &
\end{aligned}
$$

Thus, it follows from (21) that

$$
m(n+\lambda) d_{m} z^{m-1}+\cdots \equiv 0
$$

that is, $m(n+\lambda) d_{m} \equiv 0$. Since $n, s_{j}, d \in \mathbb{N}_{+}$and $d_{m} \neq 0$, we have $m=0$, that is, $\omega(z)$ is a constant. Hence, $h$ is constant, a contradiction.

From Case 1 and Case 2, we complete the proof of Theorem 1.7.

\subsection{The proof of theorem $\mathbf{1 . 8}$}

From the assumptions of Theorem 1.8, we have $E_{k}(1 ; F(z))=E_{k}(1 ; G(z))$. Thus, it follows from Lemmas 2.11 and 2.12 that $\rho(f)=\rho(g)=\rho(F)=\rho(G)$ and $S(r, F)=S(r, G)=S(r, f)=S(r, g)$.

(I) $k \geq 3$. Since

$$
\begin{aligned}
\bar{N}\left(r, \frac{1}{F(z)-1}\right) & +\bar{N}\left(r, \frac{1}{G(z)-1}\right)+\bar{N}_{(k+1}\left(r, \frac{1}{F(z)-1}\right) \\
& +\bar{N}_{(k+1}\left(r, \frac{1}{G(z)-1}\right)-N_{11}\left(r, \frac{1}{F(z)-1}\right) \\
& \leq \frac{1}{2} N\left(r, \frac{1}{F(z)-1}\right)+\frac{1}{2} N\left(r, \frac{1}{G(z)-1}\right) \\
& +S(r, F)+S(r, G) \\
& \leq \frac{1}{2} T(r, F)+\frac{1}{2} T(r, G)+S(r, F)+S(r, G) .
\end{aligned}
$$


Case 1. Suppose that $F(z), G(z)$ satisfy Lemma 2.8(i). From (10), (11) and Lemma 2.11, we have

$$
\begin{aligned}
(n+\lambda)[T(r, f)+T(r, g)] \leq & 2\left(\Gamma_{0}+\lambda\right)[T(r, f)+T(r, g)] \\
+ & o\left(\frac{T(r, f)}{r^{\delta}}\right)+o\left(\frac{T(r, g)}{r^{\delta}}\right) \\
& +o\left(\frac{T(r, f)}{r^{1-\varepsilon}}\right)+o\left(\frac{T(r, g)}{r^{1-\varepsilon}}\right)+S(r, f)+S(r, g),
\end{aligned}
$$

that is,

$$
\begin{aligned}
\left(n-2 \Gamma_{0}-\lambda\right)[T(r, f)+T(r, g)] & \leq o\left(\frac{T(r, f)}{r^{\delta}}\right)+o\left(\frac{T(r, g)}{r^{\delta}}\right)+o\left(\frac{T(r, f)}{r^{1-\varepsilon}}\right) \\
& +o\left(\frac{T(r, g)}{r^{1-\varepsilon}}\right)+S(r, f)+S(r, g) .
\end{aligned}
$$

Since $n>2 \Gamma_{0}+\lambda$ and $f, g$ are transcendental, a contradiction.

Case 2. If $F(z), G(z)$ satisfy Lemma 2.8(ii), that is,

$$
F=\frac{(b+1) G+(a-b-1)}{b G+(a-b)},
$$

where $a(\neq 0), b$ are two constants.

We now will consider three subcases as follows.

Subcase 2.1. $b \neq 0,-1$. If $a-b-1 \neq 0$, then by (23) we know

$$
\bar{N}\left(r, \frac{1}{G+\frac{a-b-1}{b+1}}\right)=\bar{N}\left(r, \frac{1}{F}\right) .
$$

Since $f, g$ are entire functions of finite order, by the second fundamental theorem, we have

$$
\begin{aligned}
T(r, G) \leq & \bar{N}\left(r, \frac{1}{G}\right)+\bar{N}\left(r, \frac{1}{G+\frac{a-b-1}{b+1}}\right)+S(r, g) \\
\leq & \bar{N}\left(r, \frac{1}{G}\right)+\bar{N}\left(r, \frac{1}{F}\right)+S(r, g) \\
\leq & \left(\Gamma_{0}+d\right)[T(r, g)+T(r, f)]+o\left(\frac{T(r, f)}{r^{\delta}}\right)+o\left(\frac{T(r, g)}{r^{\delta}}\right) \\
& +S(r, f)+S(r, g) .
\end{aligned}
$$


Then from (6) and (7), we have

$$
\begin{aligned}
\left(n+\lambda-\Gamma_{0}-d\right) T(r, g) \leq & \left(\Gamma_{0}+d\right) T(r, f)+o\left(\frac{T(r, f)}{r^{\delta}}\right)+o\left(\frac{T(r, g)}{r^{\delta}}\right) \\
& +o\left(\frac{T(r, f)}{r^{1-\varepsilon}}\right)+o\left(\frac{T(r, g)}{r^{1-\varepsilon}}\right)+S(r, f)+S(r, g) .
\end{aligned}
$$

Similarly, we have

$$
\begin{aligned}
\left(n+\lambda-\Gamma_{0}-d\right) T(r, f) \leq & \left(\Gamma_{0}+d\right) T(r, g)+o\left(\frac{T(r, f)}{r^{\delta}}\right)+o\left(\frac{T(r, g)}{r^{\delta}}\right) \\
& +o\left(\frac{T(r, f)}{r^{1-\varepsilon}}\right)+o\left(\frac{T(r, g)}{r^{1-\varepsilon}}\right)+S(r, f)+S(r, g) .
\end{aligned}
$$

From the definitions of $\Gamma_{0}$ and $\Gamma_{1}$, and since $n>2 \Gamma_{1}+\lambda$, we have $n+\lambda-$ $2 \Gamma_{0}-2 d>2\left(\Gamma_{1}-\Gamma_{0}\right)+2(\lambda-d)>0$. From the above two inequalities, we have

$$
\left(n+\lambda-2 \Gamma_{0}-2 d\right)[T(r, f)+T(r, g)] \leq S(r, f)+S(r, g),
$$

which is a contradiction with $f, g$ are transcendental.

If $a-b-1=0$, then by (23) we know $F=((b+1) G) /(b G+1)$. Since $f, g$ are entire functions, we get that $-\frac{1}{b}$ is a Picard's exceptional value of $G(z)$. By the second fundamental theorem, we have

$$
\begin{aligned}
T(r, G) & \leq \bar{N}\left(r, \frac{1}{G}\right)+S(r, G) \\
& \leq\left(\Gamma_{0}+d\right) T(r, g)+o\left(\frac{T(r, g)}{r^{\delta}}\right)+o\left(\frac{T(r, g)}{r^{1-\varepsilon}}\right)+S(r, g) .
\end{aligned}
$$

Then, from (7) and $n>2 \Gamma_{1}+\lambda$, we know $T(r, g) \leq S(r, g)$, a contradiction.

Subcase 2.2. $b=-1$. Then (23) becomes $F=a /(a+1-G)$.

If $a+1 \neq 0$, then $a+1$ is a Picard's exceptional value of $G$. Similar as the discussion as in Subcase 2.1, we can deduce a contradiction again.

If $a+1=0$, then $F G \equiv 1$, that is,

$$
P(f) \prod_{j=1}^{d} f\left(z+c_{j}\right)^{s_{j}} P(g) \prod_{j=1}^{d} g\left(z+c_{j}\right)^{s_{j}} \equiv 1 .
$$

Since $n>2 \Gamma_{1}+\lambda$, by using the same argument as in Case 1 of Theorem 1.7, we can get that the conclusion (ii) of Theorem 1.8 holds.

Subcase 2.3. $b=0$. Then (23) becomes $F=(G+a-1) / a$. 
If $a-1 \neq 0$, then $\bar{N}\left(r, \frac{1}{G+a-1}\right)=\bar{N}\left(r, \frac{1}{F}\right)$. Similar to discuss as in Subcase 2.1, we can deduce a contradiction again.

If $a-1=0$, then $F \equiv G$, that is

$$
P(f) \prod_{j=1}^{d} f\left(z+c_{j}\right)^{s_{j}} \equiv P(g) \prod_{j=1}^{d} g\left(z+c_{j}\right)^{s_{j}} .
$$

Using the same argument as in the proof of Case 2 in Theorem 1.7, we can get that $f, g$ satisfy Theorem 1.8(i).

(II) $k=2$. Since

$$
\begin{aligned}
\bar{N}\left(r, \frac{1}{F-1}\right)+ & \bar{N}( \\
& \left.r, \frac{1}{G-1}\right)-N_{11}\left(r, \frac{1}{F-1}\right) \\
& +\frac{1}{2} \bar{N}_{(k+1}\left(r, \frac{1}{F-1}\right)+\frac{1}{2} \bar{N}_{(k+1}\left(r, \frac{1}{G-1}\right) \\
& \leq \frac{1}{2} N\left(r, \frac{1}{F-1}\right)+\frac{1}{2} N\left(r, \frac{1}{G-1}\right) \\
& \leq \frac{1}{2} T(r, F)+\frac{1}{2} T(r, G)+S(r, F)+S(r, G) \\
\bar{N}_{(k+1}\left(r, \frac{1}{F-1}\right) \leq & \frac{1}{2} N\left(r, \frac{F}{F^{\prime}}\right)=\frac{1}{2} N\left(r, \frac{F^{\prime}}{F}\right)+S(r, F) \\
\leq & \frac{1}{2} \bar{N}\left(r, \frac{1}{F}\right)+S(r, F) \\
\leq & \frac{1}{2}\left(\Gamma_{0}+d\right) T(r, f)+o\left(\frac{T(r, f)}{r^{\delta}}\right)+o\left(\frac{T(r, f)}{r^{1-\varepsilon}}\right)+S(r, f)
\end{aligned}
$$

and

$\bar{N}_{(k+1}\left(r, \frac{1}{G-1}\right) \leq \frac{1}{2}\left(\Gamma_{0}+d\right) T(r, g)+o\left(\frac{T(r, g)}{r^{\delta}}\right)+o\left(\frac{T(r, g)}{r^{1-\varepsilon}}\right)+S(r, g)$.

Case 1. If $F(z), G(z)$ satisfy Lemma 2.8(i), from $f(z), g(z)$ are transcendental entire functions and (24)-(26), we have

$$
\begin{aligned}
T(r, F(z))+T(r, G(z)) \leq & 2 N_{2}\left(r, \frac{1}{F}\right)+2 N_{2}\left(r, \frac{1}{G}\right)+\left(\Gamma_{0}+d\right)[T(r, f) \\
& +T(r, g)]+o\left(\frac{T(r, f)}{r^{1-\varepsilon}}\right)+o\left(\frac{T(r, g)}{r^{1-\varepsilon}}\right) \\
& +S(r, f)+S(r, g) .
\end{aligned}
$$

Rev.Mate.Teor.Aplic. (ISSN 1409-2433) Vol. 22(2): 223-254, July 2015 
From (10), (11) and Lemma 2.11, we have

$$
\left(n-\lambda-\Gamma_{0}-2 \Gamma_{1}-d\right)[T(r, f)+T(r, g)] \leq S(r, f)+S(r, g) .
$$

Since $n>2 \Gamma_{1}+\Gamma_{0}+\lambda+d$ and $f, g$ are transcendental entire functions, we can get a contradiction.

Case 2. If $F(z), G(z)$ satisfy Lemma 2.8(ii). Similar to the proof of Case 2 in (I), we can get the conclusions of Theorem 1.8.

(III) $k=1$. Since

$$
\begin{aligned}
& \bar{N}\left(r, \frac{1}{F-1}\right)+\bar{N}\left(r, \frac{1}{G-1}\right)-N_{11}\left(r, \frac{1}{F-1}\right) \\
& \quad \leq \frac{1}{2} N\left(r, \frac{1}{F-1}\right)+\frac{1}{2} N\left(r, \frac{1}{G-1}\right) \\
& \quad \leq \frac{1}{2} T(r, F)+\frac{1}{2} T(r, G)+S(r, F)+S(r, G),
\end{aligned}
$$

we have

$$
\begin{aligned}
\bar{N}_{(2}\left(r, \frac{1}{F}\right) & \leq N\left(r, \frac{F}{F^{\prime}}\right)=N\left(r, \frac{F^{\prime}}{F}\right)+S(r, f) \leq \bar{N}\left(r, \frac{1}{F}\right)+S(r, f) \\
& \leq\left(\Gamma_{0}+d\right) T(r, f)+o\left(\frac{T(r, f)}{r^{\delta}}\right)+o\left(\frac{T(r, f)}{r^{1-\varepsilon}}\right)+S(r, f),
\end{aligned}
$$

and

$$
\bar{N}_{(2}\left(r, \frac{1}{G}\right) \leq\left(\Gamma_{0}+d\right) T(r, g)+o\left(\frac{T(r, g)}{r^{\delta}}\right)+o\left(\frac{T(r, g)}{r^{1-\varepsilon}}\right)+S(r, g) .
$$

Case 1. If $F(z), G(z)$ satisfy Lemma 2.8(i), since $f, g$ are entire functions, from (10), (11), (24)-(26), (29) and (30), we have

$$
\begin{aligned}
T(r, F)+T(r, G) \leq & 2\left(\Gamma_{1}+\Gamma_{0}+\lambda+d\right)[T(r, f)+T(r, g)] \\
& +o\left(\frac{T(r, f)}{r^{\delta}}\right)+o\left(\frac{T(r, g)}{r^{\delta}}\right)+o\left(\frac{T(r, f)}{r^{1-\varepsilon}}\right) \\
& +o\left(\frac{T(r, g)}{r^{1-\varepsilon}}\right)+S(r, f)+S(r, g) .
\end{aligned}
$$

From Lemma 2.11, we have

$$
\left[n-\lambda-2 \Gamma_{0}-2 \Gamma_{1}-2 d\right][T(r, f)+T(r, g)] \leq S(r, f)+S(r, g) .
$$


Since $n>2 \Gamma_{1}+2 \Gamma_{0}+2 d+\lambda$, from (27) and $f, g$ are transcendental, we can get a contradiction.

Case 2. If $F(z), G(z)$ satisfy Lemma 2.8(ii). Similar to the proof of Case 2 in (I), we can get the conclusions of Theorem 1.8.

(IV) $k=0$, that is, $F(z), G(z)$ share $1 I M$. From the definitions of $F(z), G(z)$, we have

$$
\begin{aligned}
\bar{N}_{L}\left(r, \frac{1}{F-1}\right) & \leq N\left(r, \frac{F}{F^{\prime}}\right)=N\left(r, \frac{F^{\prime}}{F}\right)+S(r, F) \\
& \leq \bar{N}\left(r, \frac{1}{F}\right)+S(r, F) \\
& \leq\left(\Gamma_{0}+d\right) T(r, f)+o\left(\frac{T(r, f)}{r^{\delta}}\right)+S(r, f),
\end{aligned}
$$

similarly, we have

$$
\bar{N}_{L}\left(r, \frac{1}{G-1}\right) \leq\left(\Gamma_{0}+d\right) T(r, g)+o\left(\frac{T(r, g)}{r^{\delta}}\right)+S(r, g) .
$$

Case 1. Suppose that $F(z), G(z)$ satisfy Lemma 2.9(i). From (32) and (33), we have

$$
\begin{aligned}
T(r, F(z)) \leq & N_{2}\left(r, \frac{1}{F}\right)+N_{2}\left(r, \frac{1}{G}\right)+2\left(\Gamma_{0}+d\right)[2 T(r, f)+T(r, g)] \\
& +o\left(\frac{T(r, f)}{r^{\delta}}\right)+o\left(\frac{T(r, g)}{r^{\delta}}\right)+o\left(\frac{T(r, f)}{r^{1-\varepsilon}}\right)+o\left(\frac{T(r, g)}{r^{1-\varepsilon}}\right) \\
& +S(r, f)+S(r, g) .
\end{aligned}
$$

From (10), (11) and Lemma 2.11, we have

$$
\begin{aligned}
(n+\lambda) T(r, f) \leq & \left(2 \Gamma_{0}+\Gamma_{1}+\lambda+2 d\right) T(r, f)+\left(\Gamma_{0}+\Gamma_{1}+\lambda+d\right) T(r, g) \\
& +o\left(\frac{T(r, f)}{r^{\delta}}\right)+o\left(\frac{T(r, g)}{r^{\delta}}\right)+o\left(\frac{T(r, f)}{r^{1-\varepsilon}}\right)+o\left(\frac{T(r, g)}{r^{1-\varepsilon}}\right) \\
& +S(r, f) .
\end{aligned}
$$

Similarly, we have

$$
\begin{aligned}
(n+\lambda) T(r, g) \leq & \left(2 \Gamma_{0}+\Gamma_{1}+\lambda+2 d\right) T(r, g)+\left(\Gamma_{0}+\Gamma_{1}+\lambda+d\right) T(r, f) \\
& +o\left(\frac{T(r, f)}{r^{\delta}}\right)+o\left(\frac{T(r, g)}{r^{\delta}}\right)+o\left(\frac{T(r, f)}{r^{1-\varepsilon}}\right)+o\left(\frac{T(r, g)}{r^{1-\varepsilon}}\right) \\
& +S(r, f) .
\end{aligned}
$$


From (34) and (35), we have

$$
\left(n-\lambda-3 \Gamma_{0}-3 d-2 \Gamma_{1}\right)[T(r, f)+T(r, g)] \leq S(r, f)+S(r, g) .
$$

Since $n>3 \Gamma_{0}+2 \Gamma_{1}+\lambda+3 d$, we can get a contradiction.

Case 2. Suppose that $F(z), G(z)$ satisfy Lemma 2.9(ii). Similar to the proof of Case 2 in (I), we can get the conclusions of Theorem 1.8 easily.

Thus, the proof of Theorem 1.8 is completed.

\section{Proofs of theorems 1.9 and 1.10}

\subsection{The proof of theorem 1.9}

Since $f, g$ are entire functions of finite order, from the assumptions of Theorem 1.9, by Lemma 2.12, we have $\rho(f)=\rho(g)=\rho\left(F_{1}\right)=\rho\left(G_{1}\right)$. Now, we consider three cases as follows.

Case 1. Suppose that $F_{1}$ is a Möbius transformation of $G_{1}$. Thus, it follows from the standard Valiron-Mokhon'ko lemma that

$$
T\left(r, P(f) \prod_{j=1}^{d} f\left(z+c_{j}\right)^{s_{j}}\right)=T\left(r, P(g) \prod_{j=1}^{d} g\left(z+c_{j}\right)^{s_{j}}\right)+O(\log r) .
$$

From (6), (7), Lemmas 2.4, 2.12 and the condition that $f, g$ are transcendental entire functions we deduce that there exists a subset $E \subset \mathbb{R}^{+}$with $m e s E=+\infty$ such that

$$
T(r, f) \geq r^{\rho(f)-1+\varepsilon}, T(r, g) \geq r^{\rho(g)-1+\varepsilon}, \quad r \rightarrow \infty, r \notin E,
$$

and moreover,

$$
\lim _{r \rightarrow \infty, r \notin E} \frac{T(r, f)}{T(r, g)}=1, \quad \lim _{r \rightarrow \infty, r \notin E} \frac{T(r, F)}{T(r, f)}=n+\lambda .
$$

Since $f, g$ are entire functions of finite order and $P_{0}(z)$ is a polynomial, it follows by Lemma 2.3 that

$$
\begin{aligned}
& \bar{N}\left(r, F_{1}\right)+\bar{N}\left(r, \frac{1}{F_{1}}\right) \leq \bar{N}\left(r, \frac{1}{P(f)}\right)+\sum_{j=1}^{d} \bar{N}\left(r, \frac{1}{f\left(z+c_{j}\right)}\right)+O(\log r) \\
& \quad \leq \Gamma_{0} T(r, f)+\sum_{j=1}^{d} T\left(r, \frac{1}{f\left(z+c_{j}\right)}\right)+O(\log r) \\
& \quad \leq\left(\Gamma_{0}+d\right) T(r, f)+o\left(\frac{T(r, f)}{r^{\delta}}\right)+o\left(\frac{T(r, f)}{r^{1-\varepsilon}}\right)+O(\log r),
\end{aligned}
$$


as $r \rightarrow \infty, r \notin E$, that is,

$$
\bar{N}\left(r, F_{1}\right)+\bar{N}\left(r, \frac{1}{F_{1}}\right) \leq\left(\Gamma_{0}+d\right) T(r, f)+o\left(\frac{T(r, f)}{r^{\delta}}\right)+o\left(\frac{T(r, f)}{r^{1-\varepsilon}}\right)+O(\log r) .
$$

Similarly, we have

$\bar{N}\left(r, G_{1}\right)+\bar{N}\left(r, \frac{1}{G_{1}}\right) \leq\left(\Gamma_{0}+d\right) T(r, g)+o\left(\frac{T(r, g)}{r^{\delta}}\right)+o\left(\frac{T(r, g)}{r^{1-\varepsilon}}\right)+O(\log r)$.

Thus, it follows from (37)-(40) that

$$
\bar{N}\left(r, F_{1}\right)+\bar{N}\left(r, \frac{1}{F_{1}}\right)+\bar{N}\left(r, G_{1}\right)+\bar{N}\left(r, \frac{1}{G_{1}}\right) \leq\left(\frac{2\left(\Gamma_{0}+d\right)}{n+\lambda}+o(1)\right) T\left(r, F_{1}(z)\right),
$$

as $r \rightarrow \infty, r \notin E$. By the second fundamental theorem, we have

$$
\begin{aligned}
T\left(r, F_{1}(z)\right) \leq & \bar{N}\left(r, F_{1}(z)\right)+\bar{N}\left(r, \frac{1}{F_{1}(z)}\right)+\bar{N}\left(r, \frac{1}{F_{1}(z)-1}\right)+O(\log r) \\
\leq & \left(\Gamma_{0}+d\right) T(r, f)+\bar{N}\left(r, \frac{1}{F_{1}(z)-1}\right)+o\left(\frac{T(r, f)}{r^{\delta}}\right) \\
& +o\left(\frac{T(r, f)}{r^{1-\varepsilon}}\right)+O(\log r),
\end{aligned}
$$

as $r \rightarrow \infty, r \notin E$. Thus, it follows from (6), (37) and (38) that

$$
\left(n+\lambda-\Gamma_{0}-d\right) T(r, f) \leq \bar{N}\left(r, \frac{1}{F_{1}(z)-1}\right)+o\{T(r, f)\}
$$

as $r \rightarrow \infty, r \notin E$. From (42) and the fact that $F_{1}, G_{1}$ share $1 C M^{*}$ we know that there exists $z_{0} \in \mathbb{C}$ such that $F_{1}\left(z_{0}\right)=G_{1}\left(z_{0}\right)=1$. Hence from (41), Lemma 2.5 and the condition $n>2 \Gamma_{0}+2 d-\lambda$ we get $F_{1} G_{1}=1$ or $F_{1}=G_{1}$. We discuss the following two subcases.

Case 1.1. Suppose that $F_{1}=G_{1}$. Then it follows

$$
P(f) \prod_{j=1}^{d} f\left(z+c_{j}\right)^{s_{j}}=P(g) \prod_{j=1}^{d} g\left(z+c_{j}\right)^{s_{j}} .
$$

By using the same argument as in Case 2 in the proof of Theorem 1.6, we can get the conclusion (i) of Theorem 1.9 is true.

Case 1.2. Suppose that $F_{1} G_{1}=1$, then we have

$$
P(f) \prod_{j=1}^{d} f\left(z+c_{j}\right)^{s_{j}} P(g) \prod_{j=1}^{d} g\left(z+c_{j}\right)^{s_{j}}=P_{0}(z)^{2} .
$$


We first claim from (43) that $P(z)$ has at most one zero. In fact, assuming that $P(z)$ has two zeros, say $u_{1}, u_{2}, u_{1} \neq u_{2}$, then $P(f)=a_{n}\left(f-u_{1}\right)^{n_{1}}\left(f-u_{2}\right)^{n_{2}}$, where $n_{1}, n_{2}$ are positive integers such that $n_{1}+n_{2}=n$. Since the zeros of $f-u_{1}$ and $f-u_{2}$ are also the zeros of $P_{0}(z)$, thus $f-u_{1}$ and $f-u_{2}$ have at most finitely many zeros. By using the second fundamental theorem, we can get a contradiction easily. Then we may write $P(f)=a_{n}(f-a)^{n}$, where $a$ is a complex constant. From (43), we know that $f(z), f\left(z+c_{j}\right), g(z), g\left(z+c_{j}\right),(j=$ $1,2, \ldots, d)$ have at most finitely many zeros. So, from $f, g$ are entire functions of finite order, we can write that

$$
f(z)=\varphi(z) e^{\beta(z)}+a, \quad g(z)=\psi(z) e^{\gamma(z)}+a,
$$

and

$$
f\left(z+c_{j}\right)=\varphi_{j}(z) e^{\beta_{j}(z)}, g\left(z+c_{j}\right)=\psi_{j}(z) e^{\gamma_{j}(z)},
$$

where $\varphi(z), \psi(z), \varphi_{j}(z), \psi_{j}(z)$ are nonzero polynomials, $\beta(z), \gamma(z), \beta_{j}(z)$ and $\gamma_{j}(z)$ are nonconstant polynomials for $j=1,2, \ldots, d$. From (44) and (45), we have

$$
f\left(z+c_{j}\right)=\varphi\left(z+c_{j}\right) e^{\beta\left(z+c_{j}\right)}+a \equiv \varphi_{j}(z) e^{\beta_{j}(z)} .
$$

We can get $a=0$. And since $f, g$ share $0 C M$, we have $\varphi(z)=\psi(z)$. Thus, it follows that $f=\varphi e^{\beta}$ and $g=\varphi e^{\gamma}$. Substituting them to (43), we get

$$
a_{n}^{2} \varphi^{2} \prod_{j=1}^{d} \varphi\left(z+c_{j}\right)^{2 s_{j}} \exp \left\{n(\beta+\gamma)+\sum_{j=1}^{d} s_{j}\left[\beta\left(z+c_{j}\right)+\gamma\left(z+c_{j}\right)\right]\right\} \equiv P_{0}(z)^{2}
$$

Hence, we can get that $n(\beta+\gamma)+\sum_{j=1}^{d} s_{j}\left[\beta\left(z+c_{j}\right)+\gamma\left(z+c_{j}\right)\right] \equiv(n+\lambda) \chi$ and $\beta+\gamma \equiv \chi$, where $\chi$ is a nonzero constant. Thus, it follows that

$$
a_{n}^{2} \eta \varphi(z)^{2 n} \prod_{j=1}^{d} \varphi\left(z+c_{j}\right)^{2 s_{j}} \equiv P_{0}(z)^{2}
$$

where $\eta=e^{(n+\lambda) \chi}$ is a nonzero constant.

If $\varphi(z)$ is not a constant, the degree of the left side of (46) is not less than $2(n+\lambda)$. However, since $\operatorname{deg} P_{0}<n+\lambda$, by comparing the degree of (46) on both sides, we can get a contradiction easily. Therefore, $\varphi$ and $P_{0}$ reduce to nonzero constants, say $t_{0}$ and $c$. Set $\zeta=t_{0} e^{\chi}$, then the assertion (ii) of Theorem 1.9 now follows from (46). 
Case 2. Suppose that $n>2 \Gamma_{1}+\lambda$. By (2), Lemma 2.3 and Lemma 2.11, we have

$$
\begin{aligned}
2 \bar{N}\left(r, F_{1}\right)+N_{2}\left(r \frac{1}{F_{1}}\right) \leq N_{2}\left(r, \frac{1}{P(f)}\right)+\sum_{j=1}^{d} N_{2}\left(r, \frac{1}{f\left(z+c_{j}\right)^{s_{j}}}\right) \\
\quad+O(1)+O(\log r) \\
\leq \quad\left(\Gamma_{1}+\lambda\right) T(r, f)+o\left(\frac{T(r, f)}{r^{\delta}}\right)+O(\log r) \\
\leq \frac{\Gamma_{1}+\lambda}{n+\lambda} T\left(r, F_{1}\right)+o\left(\frac{T(r, f)}{r^{1-\varepsilon}}\right)+o\left(\frac{T(r, f)}{r^{\delta}}\right)+O(\log r) \\
\leq\left(\frac{\Gamma_{1}+\lambda}{n+\lambda}+o(1)\right) T\left(r, F_{1}\right),
\end{aligned}
$$

that is,

$$
2 \bar{N}\left(r, F_{1}\right)+N_{2}\left(r \frac{1}{F_{1}}\right) \leq\left(\frac{\Gamma_{1}+\lambda}{n+\lambda}+o(1)\right) T\left(r, F_{1}\right)
$$

as $r \rightarrow \infty, r \notin E$. Similarly,

$$
2 \bar{N}\left(r, G_{1}\right)+N_{2}\left(r \frac{1}{G_{1}}\right) \leq\left(\frac{\Gamma_{1}+\lambda}{n+\lambda}+o(1)\right) T\left(r, G_{1}\right)
$$

as $r \rightarrow \infty, r \notin E$. Thus, it follows from (47), (48) and $\bar{N}\left(r, G_{1}\right)=O(\log r)$ that

$$
2 \bar{N}\left(r, F_{1}\right)+N_{2}\left(r \frac{1}{F_{1}}\right)+N_{2}\left(r \frac{1}{G_{1}}\right) \leq\left(\frac{\Gamma_{1}+\lambda}{n+\lambda}+o(1)\right) T(r)+o\{T(r)\},
$$

as $r \rightarrow \infty, r \notin E$, and where $T(r)=\max \left\{T\left(r, F_{1}\right), T\left(r, G_{1}\right)\right\}$. Since $n>$ $2 \Gamma_{1}+\lambda$, then $\frac{\Gamma_{1}+\lambda}{n+\lambda}<1$, it follows by Lemma 2.6 that $F_{1} G_{1}=1$ or $F_{1}=G_{1}$. Then by using the same argument as in Cases 1.1 and 1.2 of Theorem 1.9, we can get the conclusions.

From Cases 1 and 2, we complete the proof of Theorem 1.9.

\subsection{The proof of theorem 1.10}

By using the same argument as in proof of Theorem 1.8, we can get the conclusions of Theorem 1.10. 


\section{$5 \quad$ Proofs of theorems 1.11 and 1.12}

\subsection{The proof of theorem 1.11}

Since $f, g$ are entire functions of finite order and $\alpha(z)$ is a small function with respect to $f, g$, from the assumptions of Theorem 1.11, by Lemma 2.12, we have $\rho(f)=\rho(g)=\rho\left(F_{2}\right)=\rho\left(G_{2}\right)$. Now, we consider three cases as follows.

Case 1. Suppose that $F_{2}, G_{2}$ satisfy Lemma 2.7 (i). Since

$$
\begin{aligned}
& N_{2}\left(r, \frac{1}{F_{2}(z)}\right) \leq N_{2}\left(r, \frac{1}{P(f)}\right)+N_{2}\left(r, \frac{1}{\prod_{j=1}^{d} f\left(z+c_{j}\right)}\right)+S(r, f) \\
& \quad \leq \Gamma_{1} T(r, f(z))+\sum_{j=1}^{d} s_{j} T\left(r, f\left(z+c_{j}\right)\right)+S(r, f) \\
& \quad \leq\left(\Gamma_{1}+\lambda\right) T(r, f(z))+o\left(\frac{T(r, f(z))}{r^{\delta}}\right)+o\left(\frac{T(r, f)}{r^{1-\varepsilon}}\right)+S(r, f) .
\end{aligned}
$$

Similarly, we have

$$
N_{2}\left(r, \frac{1}{G_{2}(z)}\right) \leq\left(\Gamma_{1}+\lambda\right) T(r, g(z))+o\left(\frac{T(r, g(z))}{r^{\delta}}\right)+o\left(\frac{T(r, g)}{r^{1-\varepsilon}}\right)+S(r, g) \text {. }
$$

and

$$
N_{2}\left(r, F_{2}\right)=S(r, f), \quad N_{2}\left(r, G_{2}\right)=S(r, g) .
$$

Since $n>2 \Gamma_{1}+\lambda$, and by Lemma 2.7(i) and (50)-(52), thus we can get a contradiction with $f, g$ are transcendental entire functions.

Case 2. Suppose $F_{2}=G_{2}$, that is,

$$
P(f) \prod_{j=1}^{d} f\left(z+c_{j}\right)^{s_{j}}=P(g) \prod_{j=1}^{d} g\left(z+c_{j}\right)^{s_{j}} .
$$

By using the same argument as in Case 2 in the proof of Theorem 1.7, we can get the conclusion (i) of Theorem 1.11 is true.

Case 3. Suppose $F_{2} G_{2}=1$, that is,

$$
P(f) \prod_{j=1}^{d} f\left(z+c_{j}\right)^{s_{j}} P(g) \prod_{j=1}^{d} g\left(z+c_{j}\right)^{s_{j}}=\alpha(z)^{2} .
$$


Since $f, g$ are entire functions of finite order and $\alpha(z)$ is a small function, by comparing the zeros of both sides of (53), we get

$$
N\left(r, \frac{1}{P(f)}\right)=S(r, f), \quad N\left(r, \frac{1}{P(g)}\right)=S(r, f) .
$$

Suppose that $P(z)$ has two distinct zeros, say $\gamma_{1}, \gamma_{2}$, then $P(f)=a_{n}(f-$ $\left.\gamma_{1}\right)^{n_{1}}\left(f-\gamma_{2}\right)^{n_{2}}$, where $n_{1}, n_{2}$ are positive integers such $n_{1}+n_{2}=n$. By comparing the zeros of both sides of (53), we get

$$
N\left(r, \frac{1}{f-\gamma_{1}}\right)=S(r, f), \quad N\left(r, \frac{1}{f-\gamma_{2}}\right)=S(r, f)
$$

By using the second fundamental theorem, we can get a contradiction with $f, g$ are transcendental.

Suppose that $P(z)$ has only one zero. Thus, we can write $P(f)=a_{n}(f-$ $a)^{n}$, where $a$ is a complex constant. Since $f, g$ are entire functions of finite order and $f, g$ share $0 C M$, we can write

$$
f(z)=\phi(z) e^{\beta(z)}+a, \quad g(z)=\phi(z) e^{\gamma(z)}+a,
$$

where $\beta(z), \gamma(z)$ are nonconstant polynomials, and $\phi(z)$ is an entire function with $\rho(\phi)<\operatorname{deg} \beta, \rho(\phi)<\operatorname{deg} \gamma$. Thus, we can get $\rho(f)=\operatorname{deg} \beta$, which is a contradiction with $f$ is an entire function of finite non-positive integer order.

Thus, this completes the proof of Theorem 1.11.

\subsection{The proof of theorem 1.12}

By combining the conditions of Theorem 1.12 and using the same argument as in proof of Theorem 1.8, we can get the conclusions of Theorem 1.12.

\section{References}

[1] Banerjee, A. (2007) "Weighted sharing of a small function by a meromorphic function and its derivative", Comput. Math. Appl. 53: 1750-1761.

[2] Chen, M.R.; Chen, Z.X. (2012) "Properties of difference polynomials of entire functions with finite order", Chinese Ann. Math. 33A(3): 359-374.

[3] Chiang, Y.M.; Feng, S.J. (2008) "On the Nevanlinna characteristic of $f(z+$ $\eta)$ and difference equations in the complex plane", Ramanujan J. 16: 105129. 
[4] Fang, C.Y.; Fang, M. L. (2002) "Uniqueness of meromorphic functions and differential polynomials", Comput. Math. Appl. 44: 607-617.

[5] Fang, M.L.; Hua, X.H. (1996) "Entire functions that share one value", J. Nanjing Unvi. Math. Biquarterly 13: 44-48.

[6] Halburd, R.G.; Korhonen, R.J. (2006) "Difference analogue of the lemma on the logarithmic derivative with applications to difference equations", $J$. Math. Anal. Appl. 314: 477-487.

[7] Halburd, R.G.; Korhonen, R.J. (2006) "Nevanlinna theory for the difference operator", Ann. Acad. Sci. Fenn. Math. 31: 463-478.

[8] Halburd, R.G.; Korhonen, R.J. (2007) "Finite-order meromorphic solutions and the discrete Painleve equations", Proc. London Math. Soc. 94: 443474.

[9] Halburd, R.G.; Korhonen, R.J.; Tohge, K. (2009) "Holomorphic curves with shift-invariant hyperplane preimages", Transactions of the American Mathematical Society. Available from arXiv: 0903.3236v1. [http:// arxiv.org/abs/0903.3236v1].

[10] Hayman, W.K. (1964) Meromorphic Functions. The Clarendon Press, Oxford.

[11] Heittokangas, J.; Korhonen, R. J.; Laine, I.; Rieppo, J. (2011) "Uniqueness of meromorphic functions sharing values with their shifts", Complex Var. Elliptic Equ. 56: 81-92.

[12] Heittokangas, J.; Korhonen, R.J.; Laine, I.; Rieppo, J.; Zhang, J.L. (2009) "Value sharing results for shifts of meromorphic fucntions, and sufficient conditions for periodicity", J. Math. Anal. Appl. 355: 352-363.

[13] Ishizaki, K.; Tohge, K. (1997) "On the complex oscillation of some linear differential equations", J. Math. Anal. Appl. 206: 503-517.

[14] Lahiri, I. (2001) "Weighted sharing and uniqueness of meromorphic functions", Nagoya Math. J. 161: 193-206.

[15] Lahiri, I. (2001) "Weighted value sharing and uniqueness of meromorphic functions", Complex Var. Elliptic Equ. 46: 241-253.

[16] Laine, I. (1993) Nevanlinna Theory and Domplex Differential Equations. Walter de Gruyter, Berlin-New York. 
[17] Laine, I.; Yang, C.C. (2007) "Value distribution of difference polynomials", Proc. Japan Acad. Ser. A 83: 148-151.

[18] Li, P.; Wang, W.J. (2007) "Entire functions that share a small function with its derivative", J. Math. Anal. Appl. 328: 743-751.

[19] Li, X.M.; Li, W.L.; Yi, H.X.; Wen, Z.T. (2011) "Uniqueness theorems of entire functions whose difference polynomials share a meromorphic function of a smaller order", Ann. Polon. Math. 102: 111-127.

[20] Liu, K.; Liu, X.L.; Cao, T.B. (2012) "Some results on zeros and uniqueness of difference-differential polynomials", Appl. Math. J. Chinese Univ. 27(1): 94-104.

[21] Liu, K. (2009) "Meromorphic functions sharing a set with applications to difference equations", J. Math. Anal. Appl. 359(1): 384-393.

[22] Liu, K.; Liu, X.L.; Cao, T.B. (2011) "Value distributions and uniqueness of difference polynomials", Advances in Difference Equations 2011, Art. ID 234215, pp.12.

[23] Liu, K.; Yang, L.Z. (2009) "Value distribution of the difference operator", Arch. Math. 92: 270-278.

[24] Luo, X.D.; Lin, W.C. (2011) "Value sharing results for shifts of meromorphic functions", J. Math. Anal. Appl. 377: 441-449.

[25] Qi, X.G. (2011) "Value distribution and uniqueness of difference polynomials and entire solutions of difference equations", Ann. Polon. Math. 102: $129-142$.

[26] Qi, X.G.; Yang, L.Z.; Liu, K. (2010) “Uniqueness and periodicity of meromorphic functions concerning the difference operator", Comput. Math. Appl. 60: 1739-1746.

[27] Wang, X.L.; Xu, H.Y.; Zhan T.S. (2014) "Properties of q-shift differencedifferential polynomials of meromorphic functions", Advances in Difference Equations 2014, Art. 249, pp.16.

[28] Xu, H.Y. (2013) "On the value distribution and uniqueness of difference polynomials of meromorphic functions", Advances in Difference Equations 2013, Art. 90, pp.15. 
[29] Yang, C.C. (1972) "On deficiencies of differential polynomials", Math. Z. 125 (2): 107-112.

[30] Yang, L. (1993) Value Distribution Theory. Springer-Verlag, Berlin.

[31] Yi, H.X.; Yang, C.C. (2003) Uniqueness Theory of Meromorphic Functions. Kluwer Academic Publishers, Dordrecht; (1995) Chinese original: Science Press, Beijing.

[32] Yi, H.X. (1999) "Meromorphic functions that share one or two values II", Kodai Math. J. 22: 264-272.

[33] Zhang, J.L. (2010) "Value distribution and shared sets of differences of meromorphic functions", J. Math. Anal. Appl. 367(2): 401-408. 